\title{
Note on the Convergence Analysis of Homotopy Perturbation Method for Fractional Partial Differential Equations
}

\author{
Asma Ali Elbeleze, ${ }^{1,2}$ Adem Kılıçman, ${ }^{3}$ and Bachok M. Taib ${ }^{1}$ \\ ${ }^{1}$ Faculty of Science and Technology, Universiti Sains Islam Malaysia (USIM), 71800 Nilai, Malaysia \\ ${ }^{2}$ Department of Mathematics, Zawia University, Zawia, Libya \\ ${ }^{3}$ Department of Mathematics and Institute for Mathematical Research, University Putra Malaysia, 43400 Serdang, Selangor, Malaysia
}

Correspondence should be addressed to Adem Kılıçman; akilic@upm.edu.my

Received 7 September 2013; Revised 12 November 2013; Accepted 3 December 2013; Published 22 January 2014

Academic Editor: Hossein Jafari

Copyright (C) 2014 Asma Ali Elbeleze et al. This is an open access article distributed under the Creative Commons Attribution License, which permits unrestricted use, distribution, and reproduction in any medium, provided the original work is properly cited.

\begin{abstract}
We apply the homotopy perturbation method to obtain the solution of partial differential equations of fractional order. This method is powerful tool to find exact and approximate solution of many linear and nonlinear partial differential equations of fractional order. Convergence of the method is proved and the convergence analysis is reliable enough to estimate the maximum absolute truncated error of the series solution. The fractional derivatives are described in the Caputo sense. Some examples are presented to verify convergence hypothesis and simplicity of the method.
\end{abstract}

\section{Introduction}

Recently, the partial differential equations of fractional order have attracted much attention. This is mostly due to their frequent appearance in many applications in fluid mechanics, viscoelastic, biology, engineering, and physics $[1,2]$.

Most of partial differential equations of fractional order do not have exact analytical solution, so approximations and numerical techniques must be used. Some of these methods are series solution methods which include Adomain decomposition method [3], homotopy analysis method $[4,5]$, variationalmiteration method [6], and homotopy perturbation method [7-9]. The homotopy perturbation method [10] proposed by He in 1998. This method is useful tool for obtaining exact and approximate solution of linear and nonlinear partial differential equations of fractional order. There is no need for a small parameter or linearization, the solution procedure is very simple, and only few iterations lead to high accurate solutions which are valid for the all solution domains. The solution is expressed as the summation of an infinite series which is supposed to be convergent to the exact solution. This method has been used to solve effectively, easily, and accurately many types of fractional equations of linear and nonlinear problems with approximations. For example, [11] applied HPM to solve a class of initialboundary value problems of fractional partial differential equations over finite domain. [12] used HPM for solving the Klein-Gordon partial differential equations of fractional order. Furthermore, many authors applied HPM for solving and investigating linear and nonlinear partial differential equations of fractional ordering; see $[13,14]$. For more details about homotopy perturbation method and its applications, we refer to $[15,16]$.

Our aim in this study is to extend the applications of HPM to obtain approximate solution of some partial differential equations of fractional order such as Burgers' equation of fractional order and fractional fourth-order parabolic partial differential equation and obtain the convergence of this method.

The paper is organized as follows. In Section 2, some basic definitions and properties of fractional calculus theory are given. In Section 3, the basic idea of HPM is presented. In Section 4, analysis of HPM is given. Some examples are given in Section 5. Concluding remarks are listed in Section 6.

\section{Preliminaries}

In this section, we give some basic definitions and properties of fractional calculus theory which are used in this paper. 
Definition 1. A real function $f(x), x>0$ is said to be in space $C \mu, \mu \in R$ if there exists a real number $p>\mu$, such that $f(x)=$ $x^{p} f_{1}(x)$ where $f_{1}(x) \in C(0, \infty)$, and it is said to be in the space $C_{\mu}^{n}$ if $f^{n} \in C_{\mu}, n \in N$.

Definition 2. The Riemann-Liouville fractional integral operator of order $\alpha \geq 0$ of a function $f \in C \mu, \mu \geq-1$ is defined as

$$
J^{\alpha} f(x)=\frac{1}{\Gamma(\alpha)} \int_{0}^{x}(x-t)^{\alpha-1} f(t) d t, \quad \alpha>0, t>0
$$

in particular $J^{0} f(x)=f(x)$.

For $\beta \geq 0$ and $\gamma \geq-1$. The operator $J^{\alpha}$ has the following properties

(1) $J^{\alpha} J^{\beta} f(x)=J^{\alpha+\beta} f(x)$,

(2) $J^{\alpha} J^{\beta} f(x)=J^{\beta} J^{\alpha} f(x)$,

(3) $J^{\alpha} x^{\gamma}=\Gamma(\gamma+1) / \Gamma(\alpha+\gamma+1)^{x^{\alpha+\gamma}}$.

Definition 3. The Caputo fractional derivative of $f \in C_{-1}^{m}$, $m \in N$, is defined as

$$
\begin{array}{r}
D^{\alpha} f(x)=\frac{1}{\Gamma(m-\alpha)} \int_{0}^{x}(x-t)^{m-\alpha-1} f^{m}(t) d t, \\
m-1<\alpha \leq m .
\end{array}
$$

Lemma 4. If $m-1<\alpha \leq m, m \in N, f \in C_{\mu}^{m}, \mu>-1$, then the following two properties hold

(1) $D^{\alpha}\left[J^{\alpha} f(x)\right]=f(x)$,

(2) $J^{\alpha}\left[D^{\alpha} f(x)\right]=f(x)-\sum_{k=1}^{m-1} f^{k}(0)\left(x^{k} / k !\right)$.

\section{Homotopy Perturbation Method}

To illustrate the basic idea of this method, we consider the following nonlinear differential equation:

$$
A(u)-f(r)=0, \quad r \in \Omega
$$

with boundary conditions

$$
B\left(u, \frac{\partial u}{\partial n}\right)=0, \quad r \in \Gamma,
$$

where $A$ is a general differential operator, $B$ is a boundary operator, $f(r)$ is a known analytic function, and $\Gamma$ is the boundary of the domain $\Omega$.

In general, the operator $A$ can be divided into two parts $L$ and $N$, where $L$ is linear, while $N$ is nonlinear. Equation (3) therefor can be rewritten as follows:

$$
L(u)+N(u)-f(r)=0 .
$$

By the homotopy technique $[10,17]$ we construct a homotopy $v(r, p): \Omega \times[0,1] \rightarrow R$ which satisfies

$$
\begin{array}{r}
H(v, p)=(1-p)\left[L(v)-L\left(u_{0}\right)\right]+p[A(v)-f(r)]=0 \\
p \in[0,1], r \in \Omega
\end{array}
$$

or

$$
H(v, p)=L(v)-L\left(u_{0}\right)+p L\left(u_{0}\right)+p[N(v)-f(r)]=0,
$$

where $p \in[0,1]$ is an embedding parameter and $u_{0}$ is an initial approximation of (3) which satisfies the boundary conditions.

From (6) and (7), we have

$$
\begin{aligned}
& H(v, 0)=L(v)-L\left(u_{0}\right)=0, \\
& H(v, 1)=A(v)-f(r)=0 .
\end{aligned}
$$

The change in the process of $p$ from zero to unity is just that of $v(r, p)$ from $u_{0}(r)$ to $u(r)$. In topology, this is called deformation and $L(v)-L\left(u_{0}\right)$, and $A(v)-f(r)$ are called homotopic.

Now, assume that the solution of (6) and (7) can be expressed as

$$
v=v_{0}+p v_{1}+p^{2} v_{2}+\cdots .
$$

The approximate solution of (3) can be obtained by setting $p=1$ :

$$
u=\lim _{p \rightarrow 1} v=v_{0}+v_{1}+v_{2}+\cdots
$$

\section{Analysis on Convergence and Solution}

Consider the following fractional partial differential equations:

$$
\begin{array}{r}
{ }^{c} D_{t}^{\alpha} u(t)=f\left(t, u(t), D^{n_{1}} u(t), D^{n_{2}} u(t), \ldots, D^{n_{q}} u(t)\right), \\
t \in[0, T]
\end{array}
$$

$$
u^{k}(0)=b^{k}, \quad u(x, t)=g(x, t), \quad k=0,1,2, \ldots,
$$

where $D_{t}^{\alpha}=\partial^{\alpha} / \partial t^{\alpha}$ is Caputo fractional derivative of order $\alpha, m-1 \leq \alpha \leq m, n_{i} \in \mathbb{N}$ for every $i$. Consider that $f:[0, T] \times R \times R \times \cdots \times R \rightarrow R$ is a continuous mapping. $f\left(t, u_{1}, u_{2}, \ldots, u_{n}\right)$ exists with continuous and bounded derivatives $\partial f / \partial u_{i}$ satisfing the Lipschitz condition

$$
\begin{aligned}
& \mid f\left(t, u_{1}(t), D^{n_{1}} u_{1}(t), \ldots, D^{n_{q}} u_{1}(t)\right) \\
& \quad-f\left(t, u_{2}(t), D^{n_{1}} u_{2}(t), \ldots, D^{n_{q}} u_{2}(t)\right) \mid \\
& \quad \leq L \mid\left(u_{1}, D^{n_{1}} u_{1}, D^{n_{2}} u_{1}, \ldots, D^{n_{q}} u_{1}\right) \\
& \quad-\left(u_{2}, D^{n_{1}} u_{2}, D^{n_{2}} u_{2}, \ldots, D^{n_{q}} u_{2}\right) \mid, \quad t \geq 0,
\end{aligned}
$$

where $L$ is Lipschitz constant.

To illustrate the basic concepts of HPM for fractional partial differential equation (11) with the initial conditions (12), we construct the following homotopy for (11):

$$
\begin{gathered}
(1-p){ }_{a}^{c} D_{t}^{\alpha} u(x, t) \\
+p\left({ }_{a}^{c} D_{t}^{\alpha} u(x, t)-f\left(t, u(t), D^{n_{1}} u\right.\right. \\
\left.D^{n_{2}} u(t), \ldots, D^{n_{q}} u(t)\right)=0
\end{gathered}
$$


or

$$
\begin{aligned}
& { }_{a}^{c} D_{t}^{\alpha} u(x, t) \\
& \quad=p\left(f\left(t, u(t), D^{n_{1}} u(t), D^{n_{2}} u(t), \ldots, D^{n_{q}} u(t)\right) .\right.
\end{aligned}
$$

Substituting (9) into (15) and equating the terms with having identical power of $p$, we obtain the following series of equations:

$$
\begin{gathered}
p^{0}:{ }_{a}^{c} D_{t}^{\alpha} u_{0}(x, t)=f(x, t), \\
p^{1}:{ }_{a}^{c} D_{t}^{\alpha} u_{1}(x, t) \\
=f\left(t, u_{0}, D^{n_{1}} u_{0}(t), D^{n_{2}} u_{0}(t), \ldots, D^{n_{q}} u_{0}(t)\right), \\
p^{2}:{ }_{a}^{c} D_{t}^{\alpha} u_{2}(x, t) \\
=f\left(t, u_{1}, D^{n_{1}} u_{1}(t), D^{n_{2}} u_{1}(t), \ldots, D^{n_{q}} u_{1}(t)\right), \\
\vdots \\
p^{n}:{ }_{a}^{c} D_{t}^{\alpha} u_{n}(x, t) \\
=f\left(t, u_{n-1}, D^{n_{1}} u_{n-1}(t), D^{n_{2}} u_{n-1}(t), \ldots, D^{n_{q}} u_{n-1}(t)\right)
\end{gathered}
$$

Operating with Riemann-Liouville fractional operator $J^{\alpha}$, which is the inverse operator of Caputo derivative ${ }^{C} D_{a}^{\alpha}$ in both sides of (16) the solution

$$
\begin{gathered}
u_{0}(x, t)=\sum_{k=0}^{n-1} \frac{b^{k} t^{k}}{k !}+J^{\alpha}(f(x, t)), \\
u_{1}(x, t) \\
=J^{\alpha}\left(f\left(t, u_{0}, D^{n_{1}} u_{0}(t), D^{n_{2}} u_{0}(t), \ldots, D^{n_{q}} u_{0}(t)\right)\right) \\
u_{2}(x, t) \\
=J^{\alpha}\left(f\left(t, u_{1}, D^{n_{1}} u_{1}(t), D^{n_{2}} u_{1}(t), \ldots, D^{n_{q}} u_{1}(t)\right)\right), \\
\vdots \\
u_{n}(x, t) \quad \\
=J^{\alpha}\left(f \left(t, u_{n-1}, D^{n_{1}} u_{n-1}(t), D^{n_{2}} u_{n-1},\right.\right. \\
\left.\ldots, D^{n_{q}} u_{n-1}(t)\right)
\end{gathered}
$$

The solution of (11) in series form is given by

$$
u(x, t)=u_{1}(x, t)+u_{2}(x, t)+u_{3}(x, t)+\cdots .
$$

Define that $(C[0, T],\|\cdot\|)$ is the Banach space, the space of all continuous functions on $[0, T]$ with the norm

$$
\|f(t)\|=\max _{\forall t \in[0, T]}|f(t)| \text {. }
$$

\subsection{Existence and Uniqueness of Solutions}

Theorem 5. Let $f$ satisfy the Lipschitz condition (13) and then the problem (11) has unique solution $u(x, t)$, whenever $0<\gamma<$ 1.

Proof. Let $y$ and $z$ be two different solutions of (11), and for All $t \in[0, T]$ and $\tau \in[0, t]$ is bounded. Let $M=$ $\max _{0 \leq \tau \leq t, 0 \leq t \leq T}\left|(t-\tau)^{\alpha-1}\right|$; then,

$$
\begin{aligned}
& y-z=J^{\alpha}\left(f(t), y(t), D^{n_{1}} y(t), D^{n_{2}} y(t), \ldots, D^{n_{q}} y(t)\right) \\
& -J^{\alpha}\left(f(t), z(t), D^{n_{1}} z(t), D^{n_{2}} z(t), \ldots, D^{n_{q}} z(t)\right), \\
& y-z=\frac{1}{\Gamma(\alpha)} \int_{0}^{t}(t-\tau)^{\alpha-1} f\left(\tau, y(t), D^{n_{1}} y(t), D^{n_{2}} y\right. \\
& \left.\ldots, D^{n_{q}} y(t)\right) d \tau \\
& -\frac{1}{\Gamma(\alpha)} \int_{0}^{t}(t-\tau)^{\alpha-1} \\
& \times f\left(\tau, z(t), D^{n_{1}} z(t), D^{n_{2}} z(t), \ldots, D^{n_{q}} z(t)\right) d \tau, \\
& |y-z|=\mid \frac{1}{\Gamma(\alpha)} \int_{0}^{t}(t-\tau)^{\alpha-1} f\left(\tau, y(t), D^{n_{1}} y(t), D^{n_{2}} y,\right. \\
& \left.\ldots, D^{n_{q}} y(t)\right) d \tau \\
& -\frac{1}{\Gamma(\alpha)} \int_{0}^{t}(t-\tau)^{\alpha-1} f\left(\tau, z(t), D^{n_{1}} z,\right. \\
& D^{n_{2}} z(t), \\
& \left.\ldots, D^{n_{q}} z(t)\right) d \tau \\
& \leq \frac{L}{\Gamma(\alpha)} \int_{0}^{t}\left|(t-\tau)^{\alpha-1}\right||y-z| d \tau, \\
& \max |y-z| \leq \frac{L}{\Gamma(\alpha)} \max \int_{0}^{t}\left|(t-\tau)^{\alpha-1}\right||y-z| d \tau, \\
& \|y-z\| \leq\left[\frac{L M T}{\Gamma(\alpha)}\right]^{n} \cdot \frac{1}{n !}\|y-z\| \leq \gamma\|y-z\|, \\
& (1-\gamma)\|y-z\| \leq 0 .
\end{aligned}
$$

Since $1-\gamma \neq 0$, then $\|y-z\|=0$; therefore, $y=z$, and this completes the proof.

\subsection{Proof of the Convergence}

Theorem 6. Let $u_{n}(x, t)$ and $u(x, t)$ be defined in Banach space $(C[0, T],\|\cdot\|)$. Then the series solution $\left\{u_{n}(x, t)\right\}_{n=1}^{\infty}$ defined by (18) converges to the solution of (11), if $0<\gamma<1$. 
Proof. Suppose that $\left\{s_{n}\right\}$ is the sequence of partial sums of the series (18) and we need to show that $s_{n}(t)$ is a Cauchy sequence in Banach space $(C[0, T,\|\cdot\|])$. For this, we consider

$$
\begin{aligned}
\left\|s_{n+1}(t)-s_{n}(t)\right\| & =\left\|u_{n+1}(t)\right\| \\
& \leq \gamma\left\|u_{n}(t)\right\| \\
& \leq \gamma^{2}\left\|u_{n-1}(t)\right\| \\
& \leq \cdots \leq \gamma^{n+1}\left\|u_{0}(t)\right\| .
\end{aligned}
$$

Now, for every $n, m \in N, n \geq m$, there are two arbitrary partial sums $s_{n}$ and $s_{m}$; by using (21) and triangle inequality successively, we have

$$
\begin{aligned}
\| s_{n}- & s_{m}\|=\|\left(s_{n}(t)-s_{n-1}(t)\right) \\
& +\left(s_{n-1}(t)-s_{n-2}(t)\right)+\cdots+\left(s_{m+1}(t)-s_{m}(t)\right) \| \\
\leq & \left\|s_{n}-s_{n-1}\right\|+\left\|s_{n-1}-s_{n-2}\right\| \\
& +\cdots+\left\|s_{m+1}-s_{m}\right\| \\
\leq & {\left[\gamma^{n}+\gamma^{n-1}+\gamma^{n-2}+\cdots+\gamma^{m+1}\right] } \\
& \times\left\|u_{0}(t)\right\| \\
\leq & \gamma^{m+1}\left[\gamma^{n-m-1}+\gamma^{n-m-2}+\cdots+\gamma+1\right]\left\|u_{0}(t)\right\| \\
\leq & \gamma^{m+1}\left(\frac{1-\gamma^{n-m}}{1-\gamma}\right)\left\|u_{0}(t)\right\| .
\end{aligned}
$$

Since $0<\gamma<1$, we have $\left(1-\gamma^{n-m}\right)<1$; then,

$$
\left\|s_{n}-s_{m}\right\| \leq \frac{\gamma^{m+1}}{(1-\gamma)} \max _{\forall t \in[0, T]}\left|u_{0}(t)\right| .
$$

Since $u_{0}$ is bounded,

$$
\lim _{n, m \rightarrow \infty}\left\|s_{n}(t)-s_{m}(t)\right\|=0 .
$$

Therefore, $s_{n}(t)$ is a Cauchy sequence in $C[0, T]$, so the series converges and the proof complete.

\subsection{Error Estimate}

Theorem 7. The maximum absolute truncation error of the series solution (18) of the problem (11) is estimated to be

$$
\left|u(x, t)-\sum_{i=0}^{m} u_{i}(x, t)\right| \leq \frac{\gamma^{m+1}}{(1-\gamma)}\left\|u_{0}(t)\right\| .
$$

Proof. From Theorem 6 and inequality (22), we have

$$
\left\|u(t)-s_{m}\right\| \leq \gamma^{m+1}\left(\frac{1-\gamma^{n-m}}{1-\gamma}\right)\left\|u_{0}(t)\right\| .
$$

Since $0<\gamma<1$, we have $1-\gamma^{n-m}<1$; thus, we get the formula (25)

$$
\left|u(x, t)-\sum_{i=0}^{m} u_{i}(x, t)\right| \leq \frac{\gamma^{m+1}}{(1-\gamma)}\left\|u_{0}(t)\right\| .
$$

This completes the proof.

\section{Applications}

Example 8. Consider the following fractional partial differential equations with initial condition:

$$
\begin{gathered}
\frac{\partial^{\alpha} u}{\partial t^{\alpha}}+u \frac{\partial u}{\partial x}=\frac{\partial^{2} u}{\partial x^{2}}, \quad(x, t) \in R \times\left[0, \frac{1}{2}\right) \\
u(x, 0)=2 x
\end{gathered}
$$

with the exact solution at special case $\alpha=1$

$$
u(x, t)=\frac{2 x}{1+2 t} .
$$

To solve (28) with initial condition (29), according to the homotopy perturbation technique, we construct the following homotopy:

$$
(1-p)\left(\frac{\partial^{\alpha} u}{\partial t^{\alpha}}-\frac{\partial^{\alpha} u_{0}}{\partial t^{\alpha}}\right)=p\left(\frac{\partial^{2} u}{\partial x^{2}}-u \frac{\partial u}{\partial x}-\frac{\partial^{\alpha} u}{\partial t^{\alpha}}\right)
$$

or

$$
\frac{\partial^{\alpha} u}{\partial t^{\alpha}}-\frac{\partial^{\alpha} u_{0}}{\partial t^{\alpha}}=p\left(\frac{\partial^{2} u}{\partial x^{2}}-u \frac{\partial u}{\partial x}-\frac{\partial^{\alpha} u_{0}}{\partial t^{\alpha}}\right)
$$

Substituting of (9) in (32) and then equating the terms with same powers of $p$, we get the series

$$
\begin{gathered}
p^{0}: \frac{\partial^{\alpha} u_{0}}{\partial t^{\alpha}}=0, \\
p^{1}: \frac{\partial^{\alpha} u_{1}}{\partial t^{\alpha}}=\frac{\partial^{2} u_{0}}{\partial x^{2}}-u_{0} \frac{\partial u_{0}}{\partial x}-\frac{\partial^{\alpha} u_{0}}{\partial t^{\alpha}}, \\
p^{2}: \frac{\partial^{\alpha} u_{2}}{\partial t^{\alpha}}=\frac{\partial^{2} u_{1}}{\partial x^{2}}-u_{1} \frac{\partial u_{0}}{\partial x}-u_{0} \frac{\partial u_{1}}{\partial x}, \\
p^{3}: \frac{\partial^{\alpha} u_{3}}{\partial t^{\alpha}}=\frac{\partial^{2} u_{2}}{\partial x^{2}}-u_{2} \frac{\partial u_{0}}{\partial x}-u_{1} \frac{\partial u_{1}}{\partial x}-u_{0} \frac{\partial u_{2}}{\partial x},
\end{gathered}
$$

$$
p^{i}: \frac{\partial^{\alpha} u_{i}}{\partial t^{\alpha}}=\frac{\partial^{2} u_{i-1}}{\partial x^{2}}-\sum_{j=0}^{i-1} u_{j} \frac{\partial u_{i-j-1}}{\partial x} .
$$


Operating with Riemann-Liouville fractional operator $J^{\alpha}$, which is the inverse operator of Caputo derivative ${ }^{c} D_{a}^{a}$ in both sides of (28), the solution reads

$$
\begin{gathered}
u_{0}(x, t)=2 x, \\
u_{1}(x, t)=\frac{-4 x t^{\alpha}}{\Gamma(\alpha+1)}, \\
u_{2}(x, t)=\frac{16 x t^{2 \alpha}}{\Gamma(2 \alpha+1)}, \\
u_{3}(x, t)=\frac{-16 x t^{3 \alpha}}{\Gamma(23 \alpha+1)}\left(4+\frac{\Gamma(2 \alpha+1)}{\Gamma^{2}(\alpha+1)}\right),
\end{gathered}
$$

Since $t \leq \gamma / 2,0<\gamma<1$ and $\alpha=1$, and according to Theorem 6, we have

$$
\begin{aligned}
& \left\|s_{1}-s_{0}\right\|=\left\|\frac{-4 x t^{\alpha}}{\Gamma(\alpha+1)}\right\| \\
& =\left\|(2 x)\left(\frac{-2 t^{\alpha}}{\Gamma(\alpha+1)}\right)\right\| \\
& \leq 4\left(\frac{\gamma}{2}\right)\|2 x\|=\gamma\left\|u_{0}\right\|, \\
& \left\|s_{2}-s_{0}\right\| \leq\left\|s_{2}-s_{1}\right\|+\left\|s_{1}-s_{0}\right\| \\
& =\left\|\frac{16 x t^{2 \alpha}}{\Gamma(2 \alpha+1)}\right\|+\left\|\frac{-4 x t^{\alpha}}{\Gamma(\alpha+1)}\right\| \\
& =\left\|(2 x)\left(\frac{8 t^{2 \alpha}}{\Gamma(2 \alpha+)}\right)\right\|+\left\|(2 x)\left(\frac{-2 t^{\alpha}}{\Gamma(\alpha+1)}\right)\right\| \\
& =\|2 x\|\left(8\left(\frac{\gamma}{2}\right)^{2}+2\left(\frac{\gamma}{2}\right)\right) \\
& \leq 16\left(\frac{\gamma}{2}\right)^{2}\|2 x\|=\gamma^{2}\left\|u_{0}\right\|, \\
& \left\|s_{3}-s_{1}\right\| \leq\left\|s_{3}-s_{2}\right\|+\left\|s_{2}-s_{1}\right\| \\
& =\left\|\frac{-16 x t^{3 \alpha}}{\Gamma(3 \alpha+1)}\left(4+\frac{\Gamma(2 \alpha+1)}{\Gamma^{2}(\alpha+1)}\right)\right\|+\left\|\frac{16 x t^{2 \alpha}}{\Gamma(2 \alpha+1)}\right\| \\
& =\left\|(2 x)\left(\frac{-8 t^{3 \alpha}}{\Gamma(3 \alpha+)}\right)\left(4+\frac{\Gamma(2 \alpha)}{\Gamma^{2}(\alpha+1)}\right)\right\| \\
& +\left\|(2 x)\left(\frac{8 t^{2 \alpha}}{\Gamma(3 \alpha)}\right)\right\| \\
& \leq\left(8\left(\frac{\gamma}{2}\right)^{3}+8\left(\frac{\gamma}{2}\right)^{2}\right) \\
& \leq 32\left(\frac{\gamma}{2}\right)^{3}\|2 x\|=\gamma^{3}\left\|u_{0}\right\| .
\end{aligned}
$$

And so on, finally we get

$$
\begin{aligned}
\left\|s_{n}-s_{m}\right\| \leq & \left\|s_{n}-s_{n-1}\right\|+\left\|s_{n-1}-s_{n-2}\right\| \\
& +\left\|s_{m+1}-s_{m}\right\| \\
= & \left\|\sum_{j=0}^{n} u_{j}-\sum_{i=0}^{n-1} u_{i}\right\|+\left\|\sum_{j=0}^{n-1} u_{j}-\sum_{i=0}^{n-2} u_{i}\right\| \\
& +\cdots+\left\|\sum_{j=0}^{m+1} u_{j}-\sum_{i=0}^{m} u_{i}\right\| \\
= & \left\|u_{n}\right\|+\left\|u_{n-1}\right\|+\cdots+\left\|u_{m}\right\| \\
\leq & 2^{2 n+2}\left(\frac{\gamma}{2}\right)^{n+1}\left\|u_{0}\right\|=\gamma^{n}\left\|u_{0}\right\| .
\end{aligned}
$$

Therefore, $\lim _{n, m \rightarrow \infty}\left\|s_{n}-s_{m}\right\| \leq \lim _{n \rightarrow \infty} \gamma^{n}\left\|u_{0}\right\|=0$; that is, $s_{n}$ is, a Cauchy sequence.

Then the approximate solution in a series form is

$$
\begin{aligned}
u(x, t)= & u_{0}(x, t)+u_{1}(x, t)+u_{2}(x, t)+u_{3}(x, t)+\cdots \\
= & 2 x-\frac{4 x t^{\alpha}}{\Gamma(\alpha+1)}+\frac{16 x t^{2 \alpha}}{\Gamma(2 \alpha+1)}-\frac{16 x t^{3 \alpha}}{\Gamma(3 \alpha+1)} \\
& \times\left(4+\frac{\Gamma(2 \alpha+1)}{\Gamma^{2}(\alpha+1)}\right)+\cdots
\end{aligned}
$$

Example 9. Consider the fourth-order parabolic partial differential equation of fractional order

$$
\begin{array}{r}
\frac{\partial^{\alpha} u}{\partial^{\alpha} t}+\left(\frac{y+z}{2 \cos x}-1\right) \frac{\partial^{4} u}{\partial x^{4}}+\left(\frac{z+x}{2 \cos y}-1\right) \frac{\partial^{4} u}{\partial y^{4}} \\
+\left(\frac{x+y}{2 \cos z}-1\right) \frac{\partial^{4} u}{\partial z^{4}}=0, \quad 0<x, y, z<\frac{\pi}{3}, \\
t \in[0,1], \quad 1<\alpha \leq 2
\end{array}
$$

subject to the initial conditions

$$
\begin{aligned}
u(x, y, z, 0) & =-\frac{\partial u}{\partial t}(x, y, z, 0) \\
& =x+y+z-(\cos x+\cos y+\cos z) .
\end{aligned}
$$

The exact solution for special case $\alpha=2$ is

$$
u(x, y, z, t)=(x+y+z-\cos x-\cos y-\cos z) e^{-t} .
$$


For solving (38), according the homotopy perturbation method, we have

$$
\begin{gathered}
\frac{\partial^{\alpha} u_{0}}{\partial t^{\alpha}}=0 \\
\frac{\partial^{\alpha} u_{1}}{\partial t^{\alpha}}=-\left(\frac{y+z}{2 \cos x}-1\right) \frac{\partial^{4} u_{0}}{\partial x^{4}}-\left(\frac{z+x}{2 \cos y}-1\right) \frac{\partial^{4} u_{0}}{\partial y^{4}} \\
-\left(\frac{x+y}{2 \cos z}-1\right) \frac{\partial^{4} u_{0}}{\partial z^{4}}-\frac{\partial^{\alpha} u_{0}}{\partial^{\alpha} t} \\
\frac{\partial^{\alpha} u_{2}}{\partial t^{\alpha}=}-\left(\frac{y+z}{2 \cos x}-1\right) \frac{\partial^{4} u_{1}}{\partial x^{4}} \\
-\left(\frac{z+x}{2 \cos y}-1\right) \frac{\partial^{4} u_{1}}{\partial y^{4}}-\left(\frac{x+y}{2 \cos z}-1\right) \frac{\partial^{4} u_{1}}{\partial z^{4}}
\end{gathered}
$$

By applying $J^{\alpha}$ in both sides of (41), we got

$$
\begin{aligned}
& u_{0}(x, y, z, t) \\
& =x+y+z-(\cos x+\cos y+\cos z)(1-t), \\
& u_{1}(x, y, z, t)=x+y+z-(\cos x+\cos y+\cos z) \\
& \times\left(\frac{t^{\alpha}}{\Gamma(\alpha+1)}-\frac{t^{\alpha+1}}{\Gamma(\alpha+2)}\right), \\
& u_{1}(x, y, z, t)=x+y+z-(\cos x+\cos y+\cos z) \\
& \times\left(\frac{t^{2 \alpha}}{\Gamma(2 \alpha+1)}-\frac{t^{2 \alpha+1}}{\Gamma(2 \alpha+2)}\right), \\
& u_{n}(x, y, z, t)=x+y+z-(\cos x+\cos y+\cos z) \\
& \times\left(\frac{t^{n \alpha}}{\Gamma(n \alpha+1)}-\frac{t^{n \alpha+1}}{\Gamma(n \alpha+2)}\right)
\end{aligned}
$$

According to Theorem 6, we have

$$
\left\|s_{2}-s_{1}\right\|
$$

$$
\begin{aligned}
& \leq\left\|s_{2}-s_{1}\right\|+\left\|s_{1}-s_{0}\right\| \\
& \leq\left\|\frac{t^{2 \alpha}}{\Gamma(2 \alpha+1)}-\frac{t^{2 \alpha+1}}{\Gamma(2 \alpha+2)}\right\|+\left\|\frac{t^{\alpha}}{\Gamma(\alpha+1)}-\frac{t^{\alpha+1}}{\Gamma(\alpha+)}\right\| \\
& \leq\left\|(1-t)\left(\frac{t^{2 \alpha} / \Gamma(2 \alpha+1)-t^{(2 \alpha+1)} / \Gamma(2 \alpha+2)}{1-t}\right)\right\| \\
& +\left\|(1-t)\left(\frac{t^{\alpha} / \Gamma(\alpha+1)+t^{(\alpha+1)} / \Gamma(\alpha+2)}{1-t}\right)\right\| .
\end{aligned}
$$

But for all $t \in[0,1]$ and $\alpha=2$, we have

$$
\begin{gathered}
\left\|\frac{t^{2 \alpha} / \Gamma(2 \alpha+1)-t^{(2 \alpha+1)} / \Gamma(2 \alpha+2)}{1-t}\right\| \leq \gamma=0.0047<1 \\
\left\|\frac{t^{a} / \Gamma(\alpha+1)-t^{(\alpha+1)} / \Gamma(\alpha+2)}{1-t}\right\| \leq \gamma=0.21<1 .
\end{gathered}
$$

Thus, $\left\|s_{2}-s_{1}\right\| \leq \gamma^{2}\left\|u_{0}\right\|$

$$
\begin{aligned}
& \left\|s_{3}-s_{1}\right\| \leq\left\|s_{3}-s_{2}\right\|+\left\|s_{2}-s_{1}\right\| \\
& \leq\left\|\frac{t^{3 \alpha}}{\Gamma(3 \alpha+1)}-\frac{t^{3 \alpha+1}}{\Gamma(3 \alpha+2)}\right\|+\left\|\frac{t^{2 \alpha}}{\Gamma(2 \alpha+1)}-\frac{t^{2 \alpha+1}}{\Gamma(2 \alpha+2)}\right\| \\
& \leq\left\|(1-t)\left(\frac{t^{3 \alpha} / \Gamma(3 \alpha+1)-t^{(3 \alpha+1)} / \Gamma(3 \alpha+2)}{1-t}\right)\right\| \\
& +\left\|(1-t)\left(\frac{t^{2 \alpha} / \Gamma(2 \alpha+1)-t^{(2 \alpha+1)} / \Gamma(2 \alpha+2)}{1-t}\right)\right\| \\
& \leq\|1-t\|\left(\left\|\frac{t^{3 \alpha} / \Gamma(3 \alpha+1)-t^{(3 \alpha+1)} / \Gamma(3 \alpha+2)}{1-t}\right\|\right.
\end{aligned}
$$

Since, for all $t \in[0,1], 0<\gamma<1$, and $\alpha=2$, we have

$$
\begin{gathered}
\left\|\frac{t^{3 \alpha} / \Gamma(3 \alpha+1)-t^{(3 \alpha+1)} / \Gamma(3 \alpha+2)}{1-t}\right\| \leq \gamma=0.0004<1, \\
\left\|\frac{t^{2 \alpha} / \Gamma(2 \alpha+1)-t^{(2 \alpha+1)} / \Gamma(2 \alpha+2)}{1-t}\right\| \leq \gamma=0.0047<1 .
\end{gathered}
$$

Thus

$$
\left\|s_{3}-s_{1}\right\| \leq \gamma^{3}\left\|u_{0}\right\|
$$

where $\gamma^{3}=\left(\left(1-\gamma^{2}\right) /(1-\gamma)\right) \gamma^{2}$.

And so on, finally we get

$$
\begin{aligned}
& \left\|s_{n}-s_{m}\right\| \leq\left\|s_{n}-s_{n-1}\right\|+\left\|s_{n-1}-s_{n-2}\right\|+\cdots+\left\|s_{m+1}-s_{m}\right\| \\
& =\left\|\frac{t^{n \alpha}}{\Gamma(n \alpha+1)}-\frac{t^{n \alpha+1}}{\Gamma(n \alpha+2)}\right\| \\
& \quad+\left\|\frac{t^{(n-1) \alpha}}{\Gamma((n-1) \alpha+1)}-\frac{t^{(n-1) \alpha+1}}{\Gamma((n-1) \alpha+2)}\right\| \\
& \quad+\cdots+\left\|\frac{t^{(m+1) \alpha}}{\Gamma((m+1) \alpha+1)}-\frac{t^{(m+1) \alpha+1}}{\Gamma((m+1) \alpha+2)}\right\|
\end{aligned}
$$




$$
\begin{gathered}
\leq\left\|(1-t)\left(\frac{\left(t^{n \alpha} / \Gamma(n \alpha+1)\right)-\left(t^{n \alpha+1} / \Gamma(n \alpha+2)\right)}{1-t}\right)\right\| \begin{array}{c}
\text { The series of the solution is } \\
u(x, y, z, t)=(x+y+z-\cos x-\cos y-\cos z)
\end{array} \\
+\|(1-t)\left(\frac{\left(t^{(n-1) \alpha} / \Gamma((n-1) \alpha+1)\right)}{1-t}\right) \times\left(1-t+\frac{t^{\alpha}}{\Gamma(\alpha+1)}-\frac{t^{\alpha+1}}{\Gamma(\alpha+2)}\right. \\
\left.-\frac{\left(t^{(n-1) \alpha+1} / \Gamma((n-1) \alpha+2)\right)}{1-t}\right) \| \\
\left.+\frac{t^{2 \alpha}}{\Gamma(2 \alpha+1)}-\frac{t^{2 \alpha+1}}{\Gamma(2 \alpha+2)}+\cdots\right) .
\end{gathered}
$$$$
+\cdots+\|(1-t)\left(\frac{\left(t^{(m+1) \alpha} / \Gamma((m+1) \alpha+1)\right)}{1-t}\right.
$$$$
\left.-\frac{\left(t^{(m+1) \alpha+1} / \Gamma((m+1) \alpha+2)\right)}{1-t}\right) \|
$$$$
\leq\|1-t\|\left(\left\|\frac{\left(t^{n \alpha} / \Gamma(n \alpha+1)\right)-\left(t^{n \alpha+1} / \Gamma(n \alpha+2)\right)}{1-t}\right\|\right.
$$$$
+\| \frac{\left(t^{(n-1) \alpha} / \Gamma((n-1) \alpha+1)\right)}{1-t}
$$$$
-\frac{\left(t^{(n-1) \alpha+1} / \Gamma((n-1) \alpha+2)\right)}{1-t} \|
$$$$
+\cdots+\| \frac{\left(t^{(m+1) \alpha} / \Gamma((m+1) \alpha+1)\right)}{1-t}
$$$$
\left.-\frac{\left(t^{(m+1) \alpha+1} / \Gamma((m+1) \alpha+2)\right)}{1-t} \|\right)
$$

Since, for all $t \in[0,1], 0<\gamma<1$, and $\alpha=2$, we have

$$
\begin{aligned}
& \left\|\frac{\left(t^{n \alpha} / \Gamma(n \alpha+1)\right)-\left(t^{n \alpha+1} / \Gamma(n \alpha+2)\right)}{1-t}\right\| \leq \gamma^{n}<1, \\
& \left\|\frac{\left(t^{(n-1) \alpha} / \Gamma((n-1) \alpha+1)\right)-\left(t^{(n-1) \alpha+1} / \Gamma((n-1) \alpha+2)\right)}{1-t}\right\| \\
& \leq \gamma^{n-1}<1, \\
& \left\|\frac{t^{(m+1) \alpha}}{\Gamma((m+1) \alpha+1)}-\frac{t^{(m+1) \alpha+1}}{\Gamma((m+1) \alpha+2)}\right\| \leq \gamma^{m+1}<1 .
\end{aligned}
$$

Thus,

$$
\left\|s_{n}-s_{m}\right\| \leq \gamma^{n}\left\|u_{0}\right\|,
$$

where $\gamma^{n}=\left(\left(1-\gamma^{n-m}\right) /(1-\gamma)\right) \gamma^{m+1}$. Therefore,

$$
\lim _{n, m \rightarrow \infty}\left\|s_{n}-s_{m}\right\| \leq \gamma^{n}\left\|u_{0}\right\|=0 .
$$

That is $\left\{s_{n}\right\}$ is a Cauchy sequence.

\section{Conclusion}

In this paper, we applied the HPM for fractional partial differential equations and obtained highly approximate solutions with few iterations. Further, we introduce the study problem of convergence of HPM. The sufficient condition for convergence of this method has been presented. In this paper, we studied the convergence analysis of homotopy perturbation method for fractional partial differential equations, Further, we consider the convergence analysis of homotopy perturbation method for fractional integro-differential equations as future work.

The convergence analysis is reliable enough to estimate the maximum absolute truncation error of the series solution.

\section{Conflict of Interests}

The authors declare that there is no conflict of interests regarding the publication of this paper.

\section{Acknowledgments}

The authors are very grateful to the referees for their valuable suggestions and comments that improved the paper.

\section{References}

[1] W. R. Schneider and W. Wyss, "Fractional diffusion and wave equations," Journal of Mathematical Physics, vol. 30, no. 1, pp. 134-144, 1989.

[2] S. Momani and Z. Odibat, "Numerical comparison of methods for solving linear differential equations of fractional order," Chaos, Solitons \& Fractals, vol. 31, no. 5, pp. 1248-1255, 2007.

[3] Z. Odibat and S. Momani, "Numerical methods for nonlinear partial differential equations of fractional order," Applied Mathematical Modelling, vol. 32, no. 1, pp. 28-39, 2008.

[4] H. Jafari and S. Seifi, "Homotopy analysis method for solving linear and nonlinear fractional diffusion-wave equation," Communications in Nonlinear Science and Numerical Simulation, vol. 14, no. 5, pp. 2006-2012, 2009.

[5] H. Jafari and S. Seifi, "Solving a system of nonlinear fractional partial differential equations using homotopy analysis method," Communications in Nonlinear Science and Numerical Simulation, vol. 14, no. 5, pp. 1962-1969, 2009.

[6] S. Momani and Z. Odibat, "Comparison between the homotopy perturbation method and the variational iteration method for linear fractional partial differential equations," Computers \& Mathematics with Applications, vol. 54, no. 7-8, pp. 910-919, 2007. 
[7] S. Momani and Z. Odibat, "Homotopy perturbation method for nonlinear partial differential equations of fractional order," Physics Letters A, vol. 365, no. 5-6, pp. 345-350, 2007.

[8] O. Abdulaziz, I. Hashim, and S. Momani, "Application of homotopy-perturbation method to fractional IVPs," Journal of Computational and Applied Mathematics, vol. 216, no. 2, pp. 574-584, 2008.

[9] J. H. He, "Homotopy perturbation method: a new nonlinear analytical technique," Applied Mathematics and Computation, vol. 135, no. 1, pp. 73-79, 2003.

[10] J.-H. He, "Homotopy perturbation technique," Computer Methods in Applied Mechanics and Engineering, vol. 178, no. 3-4, pp. 257-262, 1999.

[11] A. M. A. El-Sayed, A. Elsaid, I. L. El-Kalla, and D. Hammad, "A homotopy perturbation technique for solving partial differential equations of fractional order in finite domains," Applied Mathematics and Computation, vol. 218, no. 17, pp. 8329-8340, 2012.

[12] A. M. A. El-Sayed, A. Elsaid, and D. Hammad, "A reliable treatment of homotopy perturbation method for solving the nonlinear Klein-Gordon equation of arbitrary (fractional) orders," Journal of Applied Mathematics, vol. 2012, Article ID 581481, 13 pages, 2012.

[13] A. Elsaid and D. Hammad, "A reliable treatment of homotopy perturbation method for the Sine-Gordon equation of arbitrary (fractional) order," Journal of Fractional Calculus and Applications, vol. 2, no. 1, pp. 1-8, 2012.

[14] A. K. Golmankhaneh, A. K. Golmankhaneh, and D. Baleanu, "On nonlinear fractional KleinGordon equation," Signal Processing, vol. 91, no. 3, pp. 446-451, 2011.

[15] J.-H. He, "Some asymptotic methods for strongly nonlinear equations," International Journal of Modern Physics B, vol. 20, no. 10, pp. 1141-1199, 2006.

[16] J.-H. He, "Asymptotic methods for solitary solutions and compactons," Abstract and Applied Analysis, vol. 2012, Article ID 916793, 130 pages, 2012.

[17] J.-H. He, "A coupling method of a homotopy technique and a perturbation technique for non-linear problems," International Journal of Non-Linear Mechanics, vol. 35, no. 1, pp. 37-43, 2000. 


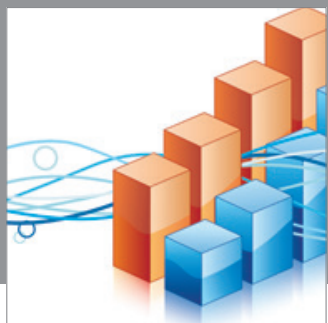

Advances in

Operations Research

mansans

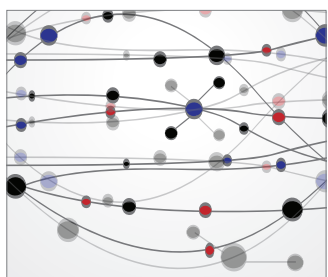

The Scientific World Journal
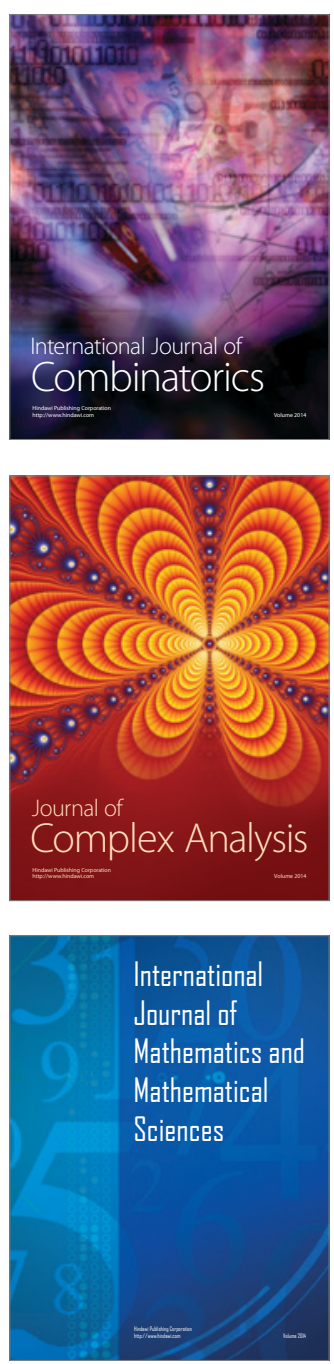
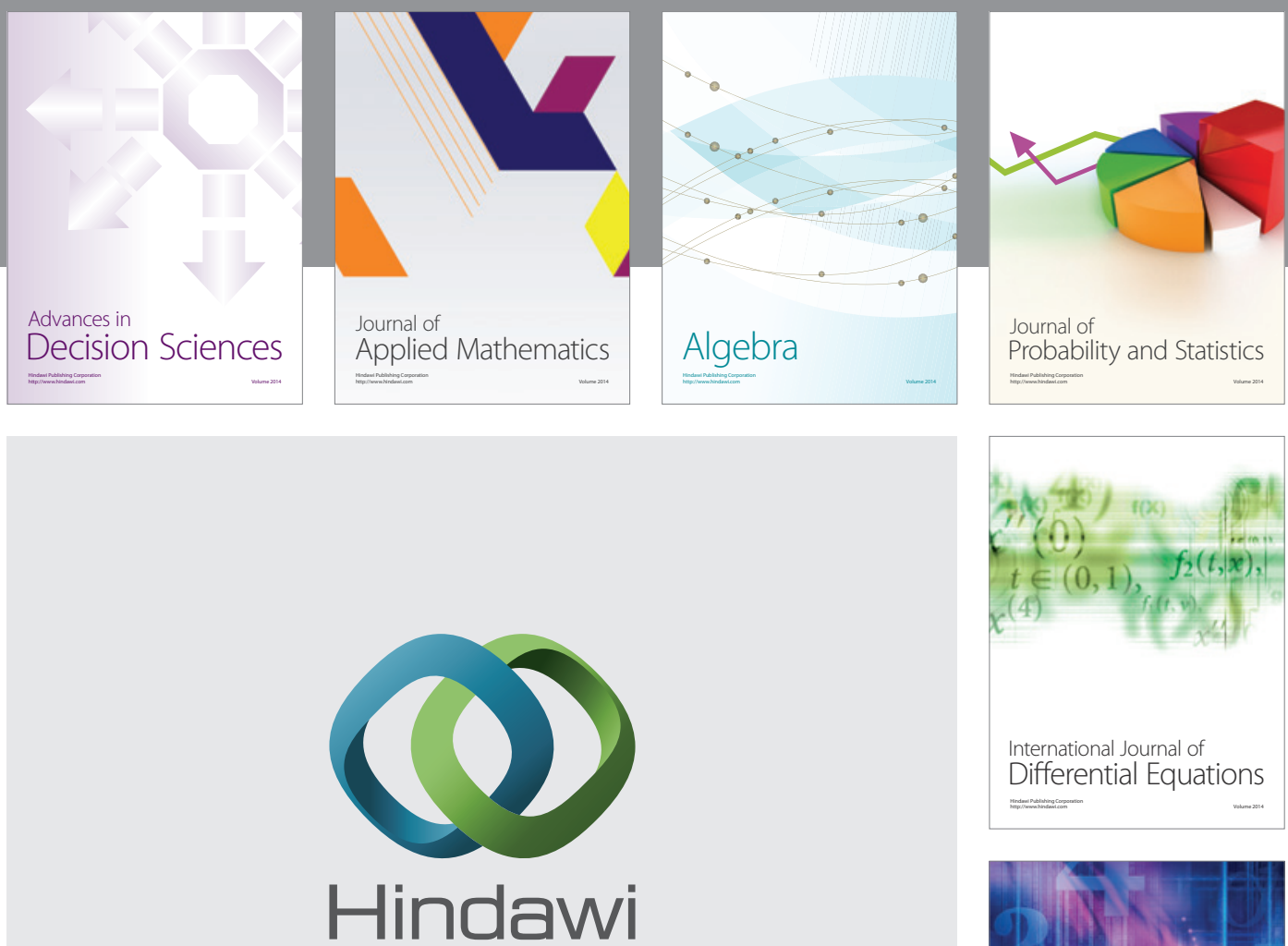

Submit your manuscripts at http://www.hindawi.com
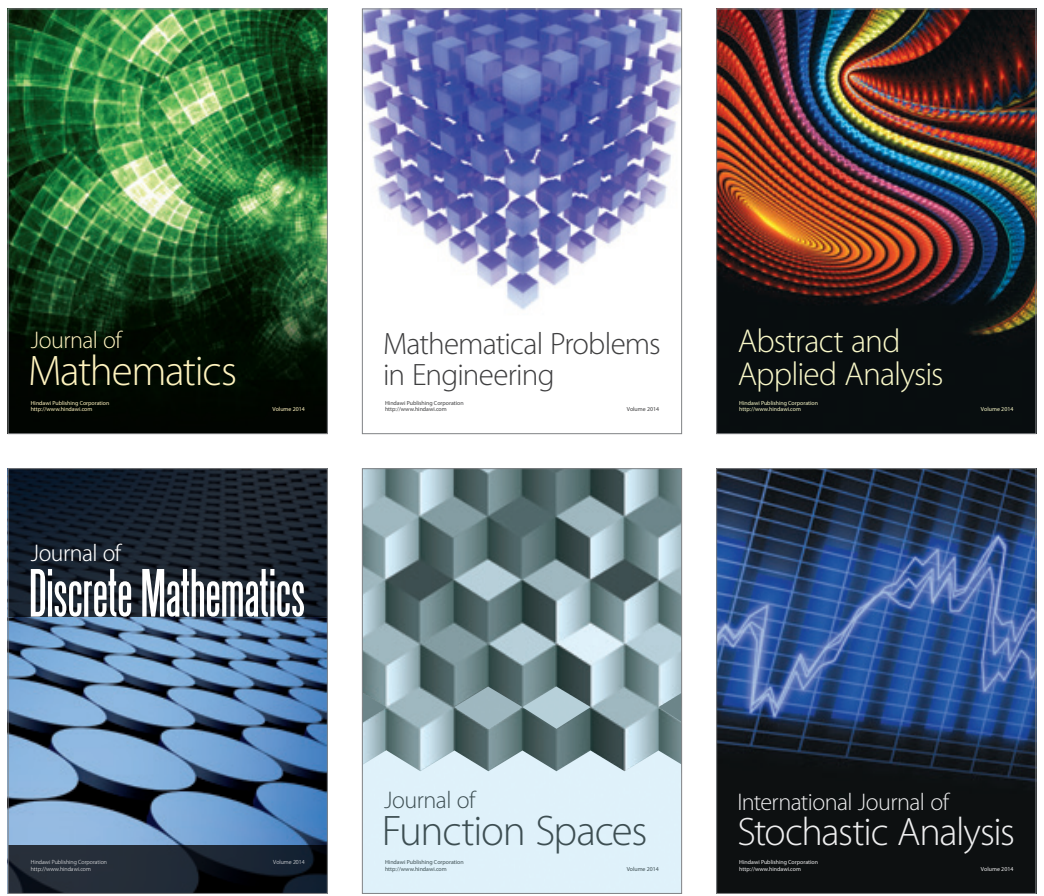

Journal of

Function Spaces

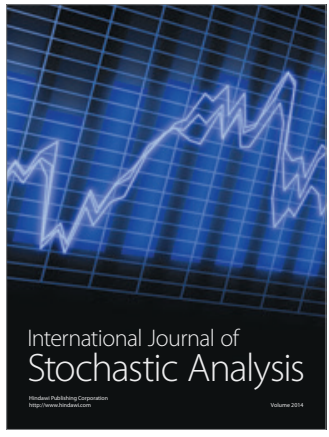

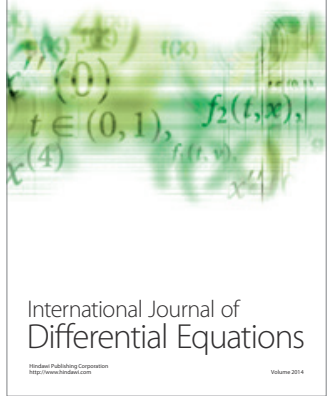
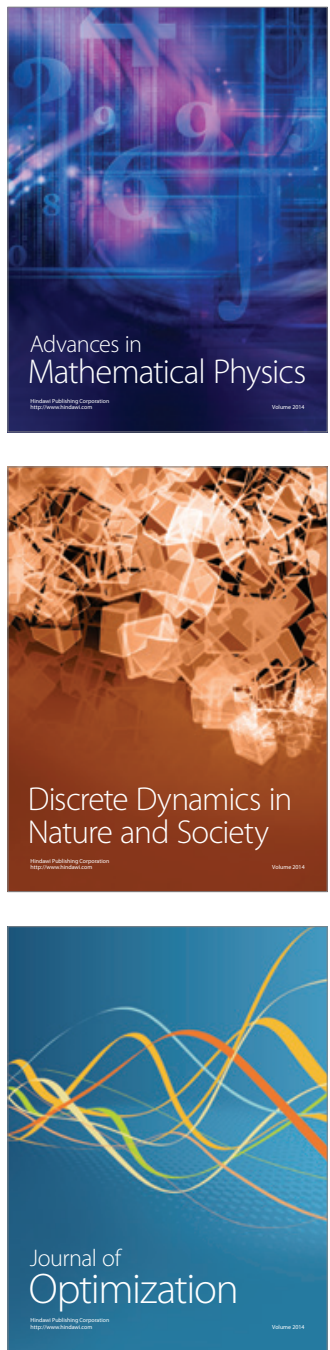